\title{
Impairment-Aware Lightpath Routing and Regenerator Placement in Optical Transport Networks With Physical-Layer Heterogeneity
}

\author{
Gangxiang Shen, Member, IEEE, Yunfeng Shen, Member, IEEE, and Harshad P. Sardesai, Member, IEEE
}

\begin{abstract}
We develop a framework that supports impairment-aware lightpath routing and wavelength assignments in optical transport networks. Different from most existing studies, we consider a more generic optical transport network with physical-layer heterogeneity, including different fiber types, variable amplification span distances and attenuation coefficients. In addition, rather than a single amplifier type as in most of the existing studies, we consider multiple amplifier types for different amplification situations. Owing to the high cost of OEO regeneration, the total number of required regenerators is considered as the major objective for optimization. A signal-quality-aware routing algorithm is developed to find routes that are expected to require the fewest regenerators. The first-fit wavelength assignment algorithm is extended to assign wavelength(s) for lightpaths after placement of some regenerators which can freely function as wavelength converters. Simulation studies indicate that the proposed algorithm can significantly reduce the required number of regenerators compared to the simple shortest-path routing algorithm. Moreover, it is found that the signal-quality-aware algorithm shows stronger benefits when a network demonstrates higher physical-layer heterogeneity such as different fiber types and non-uniform span losses. The signal-quality-aware algorithm also demonstrates better performance when a network has a higher average nodal degree. Finally, the results indicate that multiple amplifier options are important for cost-effective optical transport network design. For a network with high physical-layer heterogeneity, multiple amplifier options can significantly reduce the required number of regenerators (up to $50 \%$ ) over a single amplifier option.
\end{abstract}

Index Terms-Impairment-aware, regenerator placement, optical transport networks, physical-layer impairments, signal-quality-aware routing.

\section{INTRODUCTION}

$\mathbf{L}$ IGHTPATH routing and wavelength assignment is critical for optical transport network design [1], [2] and control plane [3]. Realizing the importance of physical-layer impairment awareness in optical network design and operation, in recent years extensive research on impairment-aware lightpath routing and wavelength assignment (IA-RWA) [4]-[11] has been performed. In the literatures, the investigations on IA-RWA mainly focus on the following directions.

Manuscript received June 24, 2010; revised February 01, 2011, April 17, 2011, July 08, 2011; accepted July 18, 2011. Date of publication July 25, 2011; date of current version September 07, 2011.

G. Shen was with Ciena Corporation, MD, USA. He is now with the School of Electronic and Information Engineering, Soochow University, Suzhou 215006, China (e-mail: shengx@ suda.edu.cn).

Y. Shen and H. P. Sardesai are with Ciena Corporation, MD 21090 USA (e-mail: yshen@ciena.com).

Digital Object Identifier 10.1109/JLT.2011.2162939
First, because $3 \mathrm{R}$ regenerator is one of the most expensive components in the optical transport networks, much effort was dedicated to efficiently choosing regeneration sites and allocating regenerators [12]-[14], [8], [7], [15]. Second, given a set of regeneration sites and regenerators, a range of lightpath routing and wavelength assignment algorithms were developed to efficiently utilize the regeneration capability, such that lightpath demands are maximally satisfied and a minimum lightpath blocking probability is achieved [12], [7], [16]-[19]. Third, in the perspective of control plane, GMPLS-based techniques were extended to support regenerator information distribution [20], [21]. These include flooding Link State Advertisement (LSA) messages that contain regenerator information via the OSPF-TE routing protocol, and exchanging signaling messages that contain regenerator information via the RSVP-TE signaling protocol.

Based on these existing studies, we find that the following two aspects on IA-RWA are yet to be further explored:

First, almost all the existing studies assume the uniformness of the physical-layer parameters [4]-[11], including: 1) the same fiber type in an entire network; 2) uniform amplification span distance; 3) uniform fiber attenuation coefficient; and 4) the same amplifier type and noise figure (NF). These assumptions are however not practical for most real optical transport networks.

An optical transport network can contain different types of fibers. They exhibit different levels of nonlinearity due to their different optical characteristics such as local dispersion, effective area, and so on. For low fiber nonlinearity, different fiber types are launched with different optical channel powers. These different launch powers impact the receiving optical signal-tonoise ratio (OSNR) of a lightpath, which further affects route selection and regenerator placement of the lightpath. Thus, it is necessary to incorporate fiber type as an important system parameter in optical transport network design.

In addition, though $80 \mathrm{~km}$ is a typical continental amplification span distance, actual span distances in an optical transport network are far from uniform. They vary over a large range. For example, the standard deviations of amplification span distances in some national optical transport networks are greater than $20 \mathrm{~km}$. In addition, due to different fiber types and ages, the fiber span attenuation coefficients are also variable. Thus, when modeling lightpath impairments, we should take into account the non-uniformness of both amplification span distance and attenuation coefficient.

Second, most of the studies route lightpaths based on the shortest paths. For an optical network with the same fiber type and uniform distributions of amplification span distance 
and span attenuation coefficient, the shortest-path algorithm is sufficient to achieve a good performance that requires the fewest regenerators, as the shortest route generally shows the best signal quality among all the routes between a pair of nodes. However, under a heterogeneous network environment, in which all the physical-layer parameters including amplification span distance, span attenuation coefficient, and fiber type are non-uniform, the shortest route does not always guarantee the best signal quality. We see many cases in real network designs, in which a long route actually shows a better signal quality than a short route. Because a route with a better signal quality in general requires fewer intermediate regenerators, it is wise to choose a route with the best signal quality. Thus, in addition to the simple shortest-path algorithm, a more advanced signal-quality-aware routing algorithm should be developed for more efficient network design.

In this paper, we develop a generic framework for impairment-aware lightpath routing and wavelength assignment which takes into account various physical-layer heterogeneity, including variable amplification span distances and attenuation coefficients, as well as different fiber types. Rather than the simple shortest path routing algorithm, we propose a new routing algorithm called the signal-quality-aware algorithm to search for a route that requires the smallest number of regenerators between a pair of nodes. If there are multiple routes that require the same smallest number of regenerators, the shortest one is selected for better wavelength capacity efficiency. We also develop an efficient regenerator placement algorithm that can minimize the number of placed regenerators on a lightpath. These placed regenerators can be reused as "free" wavelength converters in the subsequent wavelength assignments for lightpaths. Simulation studies indicate that the proposed signal-quality-aware routing algorithm consistently outperforms the simple shortest-path routing algorithm. The improvement becomes significant when a network shows strong physical-layer heterogeneity and has a high nodal degree.

The approach developed in this paper mainly targets at optical network planning; however, given the fiber layer information such as the locations of optical amplifiers, fiber span loss, and so on, the approach can also be extended to find eligible lightpath routes and wavelengths for dynamic lightpath service provisioning. In addition, in this paper we evaluate the total number of required $3 \mathrm{R}$ regenerators and wavelength converters by considering these two types of components equivalent, i.e., $3 \mathrm{R}$ regenerators can function as wavelength converters and wavelength converters are essentially OEO signal regenerators.

The rest of this paper is organized as follows. In Section II, we describe the detail on how we evaluate signal quality of a lightpath. In Section III, we introduce the signal-quality-aware routing algorithm and the regenerator placement algorithm. We also describe the step of wavelength assignment and the corresponding wavelength converter placement algorithm. In Section IV, we present and analyze simulation results. Section V concludes the paper.

\section{Physical-Layer Signal Quality Modeling AND EVALUATION}

Though there are many physical-layer impairment models that evaluate different network impairment aspects in the literatures, for simplicity in this study we consider the most important impairment in the optical transport network, namely optical amplifier amplified spontaneous emission (ASE) noise. Based on the accumulated ASE noise and the signal power on an optical channel, we find OSNR that is used to determine whether the signal quality of the optical channel is good or signal regenerators should be placed. For the other physical-layer impairment effects, such as chromatic dispersion, amplification ripple, polarization mode dispersion (PMD) and polarization dependent loss (PDL), reconfigurable optical add-drop multiplexer (ROADM) filter concatenation [22], and nonlinearity effects [23], we use a net OSNR penalty to count for their overall impact on the signal quality. The penalty value is consistently set to be $2.5 \mathrm{~dB}$ in this study. Nonetheless, such a simplified OSNR-based signal quality evaluation does not prevent us from using other more advanced impairment-aware signal quality evaluation model.

\section{A. Amplifier Selection Strategy and ASE Noise}

1) Amplifier Selection: In an optical transport network, Erbium doped fiber amplifier (EDFA) and Raman amplifier are two typical types of amplifiers. We may employ a single type of amplifier to support most span-loss situations. Such a "single type fit all" approach is however in general not efficient as span losses often vary over a wide range in real optical transport networks. An amplifier designed for high-loss compensation usually cannot best support a low-gain situation, and vice verse. To achieve a cost-effective optical transport network design, it is necessary to deploy multiple types of amplifiers, of which each optimally supports a certain gain range. It is found to be sufficiently efficient to use two or three EDFA amplifier types to support low, middle, and high gain situations, respectively. In addition, in order to achieve even better amplification performance (for lower ASE noise), Raman amplifiers can also be deployed solely or collocated with any one of the EDFA amplifier types. In this study, we employ multiple types of amplifiers, which include a 15-dB EDFA, a 22-dB EDFA, and a 7-dB Raman amplifier. The Raman amplifier works with the EDFAs. Here the value in the name of each amplifier type corresponds to the maximal operational amplification gain that can be supported by this type of amplifier. For a specific fiber span loss, rather than a maximal amplifier gain, an actual gain that compensates for this loss is applied.

Given different types of amplifiers or amplifier combinations of EDFA and Raman amplifier, a range of strategies can be applied to select amplifier types. One is to select amplifier types based on noise figure (NF). Specifically, among a set of eligible amplifier types, the one with the lowest NF for a certain gain requirement is selected. User preference can be another strategy. Each amplifier type is assigned with a certain preference priority. The one with the highest priority is selected first. If there are multiple amplifier types at the same priority level, a second criterion such as NF can be applied to break the tie. Finally, cost is another important criterion for amplifier type selection, i.e., the one with the lowest cost can be selected first. In this study, we employ the best-NF strategy for the amplifier selection.

2) NF Determination: The noise figure (NF) of an amplifier is usually not uniform in its gain range. For more accurate 


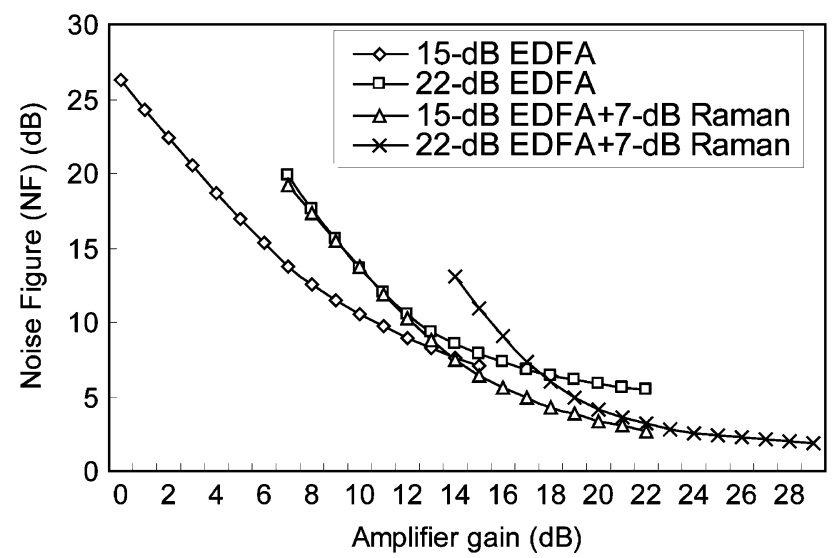

Fig. 1. Noise Figures (NF) of different types of optical amplifiers/amplifier combinations under different gains.

signal quality modeling, it is important to use an accurate NF based on the actual gain of an amplifier. Once an amplifier type is selected, NF can be determined based on a required amplification gain. We may use a mathematical formula that is specifically modeled for the amplifier type to calculate the NF; we may also find an NF by looking up a gain-NF table that is specifically pre-built for the amplifier type. In this study, we employ the second approach to find the NF values, which were obtained from the real experiments of different types of amplifiers. Fig. 1 shows the NF-gain curves of the above different types of optical amplifiers/amplifier combinations.

3) ASE Noise Calculation: When a lightpath passes an amplifier, in the literature most papers employ the following formula to calculate the ASE noise

$$
P_{\mathrm{ase}}^{o}=P_{\mathrm{ase}}^{i} G+2 n_{\mathrm{sp}}(G-1) h v_{i} B_{o}
$$

where $P_{\text {ase }}^{i}$ is the unpolarized power level of the accumulated ASE noise before a lightpath enters an amplifier, $P_{\text {ase }}^{o}$ is the power level of the accumulated ASE noise after a lightpath transverses an amplifier, $n_{\mathrm{sp}}$ is the ASE noise factor, which is gain-dependent and can be derived from NF, $h$ is Plank's constant, $v_{i}$ is the optical signal frequency, $B_{o}$ is the receiver's optical bandwidth, and $G$ is the gain of the optical amplifier. The formula is generic to model an EDFA, a Raman amplifier, or their combinations. In our calculation, based on a real industrial optical transport system, we employ the following simple equation to model the optical amplifier ASE noise

$$
P_{\text {ase }}(\mathrm{dBm})=-58(\mathrm{dBm})+G(\mathrm{~dB})+\mathrm{NF}(\mathrm{dB})
$$

where $P_{\text {ase }}(\mathrm{dBm})$ is the power of the ASE noise introduced by an optical amplifier in 0.1-nm spectrum, whose unit is $\mathrm{dBm}$, $G(\mathrm{~dB})$ is the gain of the optical amplifier in $\mathrm{dB}$, and $\mathrm{NF}(\mathrm{dB})$ is the noise figure (in $\mathrm{dB}$ ) of the optical amplifier under the current gain.

\section{B. Physical-Layer Heterogeneity}

The physical-layer heterogeneity shows a strong impact on the impairment evaluation. We consider the following major heterogeneity aspects in this study. First, a network can contain multiple fiber types, including non-dispersion shifted fiber (NDSF), enhanced large effective area fiber (ELEAF), TrueWave (TW), LEAF submarine (LS), and dispersion shifted fiber (DSF). For nonlinearity effect control, the channel launch powers in these fiber types are set to be as follows: NDSF: 0 $\mathrm{dBm}$, ELEAF: $-1 \mathrm{dBm}$, True Wave: $-2 \mathrm{dBm}, \mathrm{LS}:-3 \mathrm{dBm}$, and DSF: $-4 \mathrm{dBm}$. Second, the amplification span distances on each fiber link are variable; the fibers attenuation coefficients also vary depending on fiber types and span ages. The variances of amplification span distances and attenuation coefficients affect the required amplification gain, which further determines amplifier selection, NF, and ASE noise.

\section{IMPAIRMENT-AWARE LIGHTPATH ROUTING AND WAVELENGTH ASSIGNMENT}

\section{A. High-Level Steps of Lightpath Routing and Wavelength Assignment}

Lightpath routing and wavelength assignment has been studied for many years [1]. For efficient and fast solutions, heuristics that discompose the steps of lightpath routing and wavelength assignment are proposed in most of the existing studies. Specifically, a lightpath route is first found and wavelengths are then assigned along the route. This framework also takes such a strategy, whose major steps are as follows.

Step 1) Apply a lightpath routing algorithm to find a candidate route; different routing algorithms such as the shortest path routing, least-congested routing, and signal-quality-aware routing algorithms can be applied. We will introduce the signal-quality-aware routing algorithm later.

Step 2) For the found route, place regenerators subject to a predefined OSNR threshold. A lightpath whose net OSNR is lower than the threshold is placed with regenerators in the middle to refresh its optical signal. The detail on the regenerator placement is presented in Section III-C.

Step 3) Assign wavelength(s) on each of the links along a lightpath. The wavelength assignment process should make full use of the wavelength conversion capability of the placed regenerators (if there are any). If there are still wavelength collisions, additional wavelength converters are placed.

Due to the high cost of signal regeneration as well as wavelength conversion, minimizing the required number of signal regenerators and wavelength converters is the major optimization objective in the algorithm. In addition, as a potential future research topic, it is interesting to develop a more efficient algorithm that integrates both regenerator and wavelength converter placements. However, for simplicity this study separates the two steps.

\section{B. Signal-Quality-Aware Lightpath Routing Algorithm}

To minimize the required number of regenerators, we propose a new lightpath routing algorithm that takes into account optical channel signal quality to select a route that is expected to require 
the fewest regenerators. The steps of the algorithm are described here.

Step 1) Based on the controlled channel launch powers on different fiber types, determine OSNR for each fiber link.

Step 2) Run the shortest path routing algorithm (e.g., Dijkstra's algorithm) to find a route that has the best OSNR by considering $1 / \mathrm{OSNR}_{s}$ of each fiber link as link cost.

On each fiber link, a controlled launch power is always maintained whenever an optical signal traverses an optical amplifier. For example, for an NDSF fiber, a constant channel launch power $0 \mathrm{dBm}$ is maintained whenever an optical signal traverses an optical amplifier. To find a route with the best OSNR, we use the linear summation relationship existing between a total ONSR of a lightpath and its individual component OSNRs (i.e., link OSNRs) that contribute to the total OSNR, given as follows:

$$
1 / \mathrm{OSNR}_{\text {total }}=\sum_{s \in S} 1 / \mathrm{OSNR}_{s}
$$

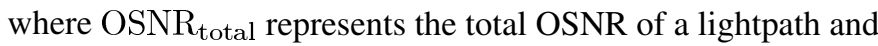
$\mathrm{OSNR}_{s}$ is the $s$ th contributor to the total OSNR, which is the OSNR on the $s$ th link of the lightpath. Here all the OSNRs are linear values (not in $\mathrm{dB}$ ).

To find a route with a minimum sum $\sum_{s \in S} 1 / \mathrm{OSNR}_{s}$, we define $1 / \mathrm{OSNR}_{s}$ of each link as its cost and apply Dijkstra's shortest-path routing algorithm to find a route that has the best OSNR.

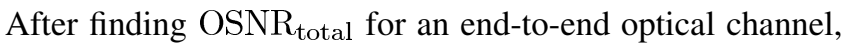
we add a $2.5-\mathrm{dB}$ net penalty to account for all the other nonASE-noise impairment effect.

Also, in order to ensure that a found route consumes the fewest wavelength sources, we make an enhancement to the signal-quality-aware routing algorithm. Specifically, in addition to finding a best-ONSR route, we find the shortest route and compare the required numbers of regenerators by the shortest route and the best-OSNR route (the regenerator placement algorithm is introduced later) to choose the one requiring fewer regenerators. If a shortest route and a best-OSNR route require the same number of regenerators, we select the shortest route due to its less wavelength resource consumption and lower end-to-end service latency.

Also, when routing lightpaths, we consider the constraint of maximal wavelength count supported by each fiber link. For example, a typical fiber communication system might maximally support 88 wavelengths on each fiber. Under heavy lightpath demand, all the wavelengths on a fiber link can be exhausted after routing some a priori services. For subsequent lightpath demand, we need to remove the congested link from the network before routing lightpaths such that any found route is ensured with sufficient free wavelength(s) on any traversed links.

\section{Regenerator Placement on a Lightpath}

The detail of regenerator placemen algorithm is straightforward. Starting from the source node of a route, we evaluate the OSNR of a lightpath when it reaches an intermediate node $\mathrm{X}$. If the OSNR is greater than a predefined threshold, we move to the next node on the route and continue the OSNR evaluation;

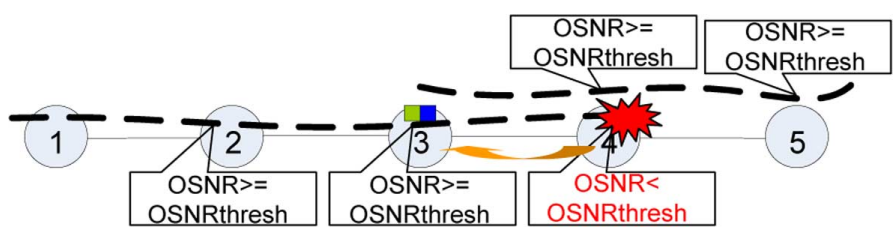

Fig. 2. Example of regenerator placement on a lightpath.

otherwise, we place a regenerator at a node just before $X$ to refresh the signal. Next, starting from the node with the placed regenerator, we repeat the same process for the remaining part of the route. The placement process is terminated when the destination node is reached. Because the algorithm maximizes optical reach before adding a new regenerator, if there are no additional wavelength converters required to resolve wavelength collision, such a regenerator placement strategy always ensures a minimum number of regenerators.

Fig. 2 shows an example for the regenerator placement algorithm. Starting from node 1, we evaluate OSNRs on nodes 2, 3 , and 4 . We find that both nodes 2 and 3 show good OSNRs and when the lightpath reaches node 4 the OSNR is lower than a threshold, which means signal regeneration is required. We move back to node 3 and place a regenerator. Next, starting from node 3 , we repeat the previous process until reaching destination node 5 .

\section{Wavelength Assignment and Converter Placement}

Wavelength assignment is another important part of IA-RWA. Many wavelength assignment algorithms were proposed, such as first-fit, max-use algorithms, and so on. Interesting readers may refer to [2] for these algorithms. The current study simply extends the first-fit algorithm for the wavelength assignment due to its popularity and good efficiency. Of course, other wavelength assignment algorithms can be applied here as well.

Different from the traditional wavelength assignments, wavelength conversion capability of the placed regenerators is an important advantage that we can take in the current study. We should maximally use the placed regenerators as wavelength converters before placing any additional wavelength converters when there are any wavelength collisions. Specifically, we divide a lightpath into segments based on the placed regenerators. If there are no regenerators, the lightpath is considered as a single segment; if there is one regenerator, the lightpath is divided into two segments with first segment containing all the links from the source node to the regenerator node and the second segment containing all the remaining links from the regenerator node to the destination node. In Fig. 2, the lightpath is made up of two segments, i.e., segment (1-2-3) and segment (3-4-5). In a general case, if a route has $N$ intermediate regenerators, it is divided into $N+1$ segments, of which each is terminated at a regenerator node. Because between any two consecutive lightpath segments there is a regenerator that is capable of wavelength conversion, we only need to ensure wavelength continuity on each segment.

We employ the first-fit algorithm [1] to assign wavelengths for each lightpath segment. A brief description on the algorithm is as follows. Given a found lightpath route, we first scan all the 


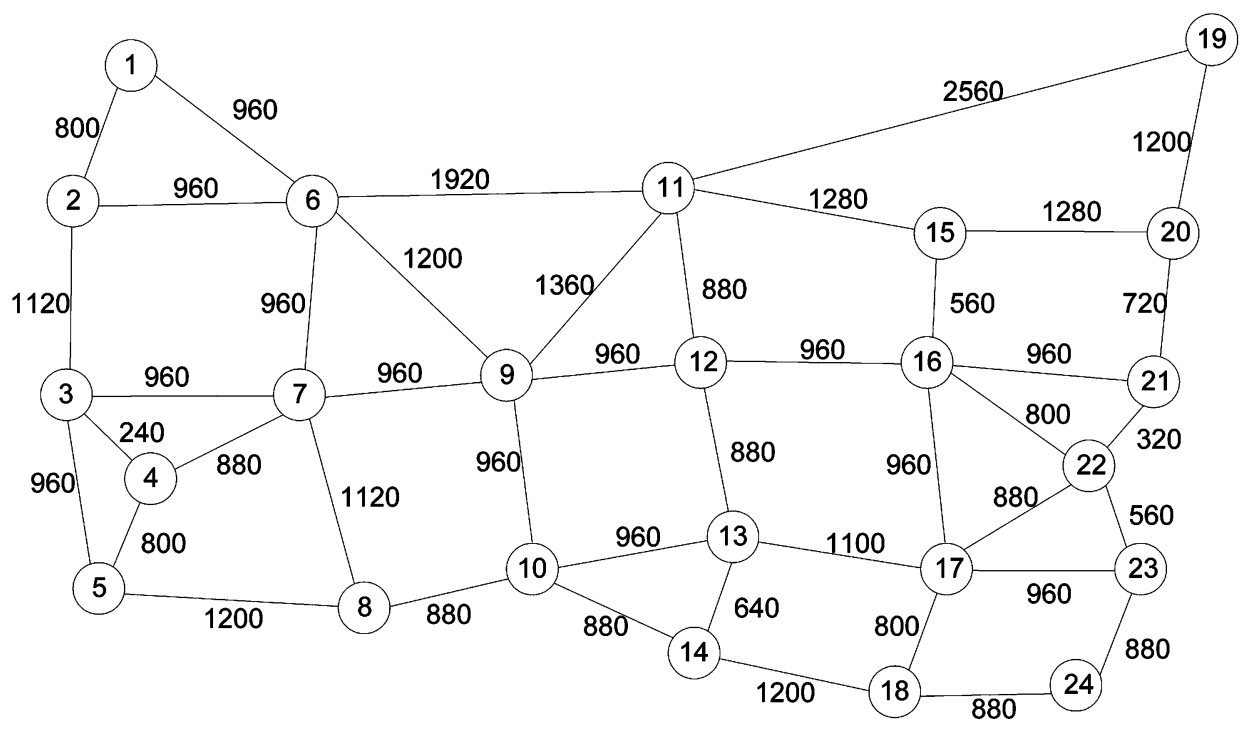

Fig. 3. Topology of USNET. The number by each link is the physical distance $(\mathrm{km})$ of the link.

fiber links on the route to find a common set of free wavelengths. Next, from this common set of free wavelengths, we select a wavelength that has the smallest index. However, if the common wavelength set is empty, which means that there is wavelength collision, we need to place wavelength converters to resolve the collision. The step of wavelength converter placement is similar to that of regenerator placement, whose steps are briefed as follows.

Step 1) Initialize a common free wavelength set X1, which contains all the free wavelengths on the first link L1 of the route.

Step 2) Consider the second link L2 on the route and generate a cross set X2 of free wavelengths on both links L1 and L2. Only a wavelength that is free both in X1 and on $\mathrm{L} 2$ can be included in X2.

Step 3) Examine cross set $\mathrm{X} 2$ to see if it is empty. If so, place a wavelength converter at the first end node of L2 and then consider L2 as the first link in Step 1 and repeat the subsequent steps. Otherwise, move to next link L3 and perform a set-crossing operation to generate cross set X3. Again, examine set X3 to see if it is empty and perform the subsequent steps accordingly. The above process is terminated when the last link on the route is examined.

\section{Simulations AND Result Analyses}

\section{A. Simulation Assumptions and Conditions}

We ran simulations for two test networks. One is a real carrier network $\mathrm{A}$, which contains more than 150 nodes and more than 170 links, and the other is USNET, which is a benchmark topology widely used for performance evaluation in the literatures [5], [11]. Fig. 3 shows the USNET network topology. Note that the length of each fiber link of the two network topologies has been normalized to the nearest value to integer times of 80 $\mathrm{km}$. For example, if a link length is $145 \mathrm{~km}$, we normalize it to be $160 \mathrm{~km}$, and if the length is $100 \mathrm{~km}$, we normalize it to be $80 \mathrm{~km}$. The purpose of this is to facilitate the control of amplification span distance variance during the simulation evaluation.

To evaluate the impact of the variances of amplification span distances and attenuation coefficients, we assume that both the amplification span distances and attenuation coefficients follow Gaussian distributions. Based on the actual statistical data obtained from the large real carrier network $\mathrm{A}$, we set the mean of span distance to be $80 \mathrm{~km}$, the mean of attenuation coefficient to be $0.225 \mathrm{~dB} / \mathrm{km}$, and the standard deviation (std.) of attenuation coefficient to be $0.0064 \mathrm{~dB} / \mathrm{km}$. To limit the fiber span distances, we set the lower and upper bounds of span distance to be $20 \mathrm{~km}$ and $140 \mathrm{~km}$, respectively. For the attenuation coefficient, the lower and upper bounds are set to be $0.2 \mathrm{~dB} / \mathrm{km}$ and $0.25 \mathrm{~dB} / \mathrm{km}$, respectively. Each ROADM is assumed to have a fixed 15-dB loss.

All the traffic demands are lightpaths. The traffic demand of the large carrier network A contains $40-\mathrm{Gb} / \mathrm{s}$ connections, groomed from real low-rate traffic $(2.5-\mathrm{Gb} / \mathrm{s}$ and $10-\mathrm{Gb} / \mathrm{s}$ subwavelength services). The sum of all end-to-end lightpath demands is 357 units. Here each unit of lightpath demand is an end-to-end optical channel with single-wavelength capacity. The demand of the USNET network contains $10-\mathrm{Gb} / \mathrm{s}$ connections, which is randomly generated by assuming each node pair has a random number, ranging from 0 to 3 , of $10-\mathrm{Gb} / \mathrm{s}$ lightpath channels. The sum of all end-to-end lightpath demands is 244 units. We also assume the networks maximally carry 88 wavelengths on each fiber.

For performance comparison, we consider the shortest path routing and the signal-quality-aware routing algorithms. For simulation purpose, the OSNR threshold is set to be $14.0 \mathrm{~dB}$ for both $10-\mathrm{Gb} / \mathrm{s}$ and $40-\mathrm{Gb} / \mathrm{s}$ lightpaths. As one of major objectives, we aim to minimize the total number of required regenerators (and wavelength converters) subject to the conditions that all the lightpath demands are provisioned and the predefined OSNR threshold is satisfied on all the lightpath segments. 


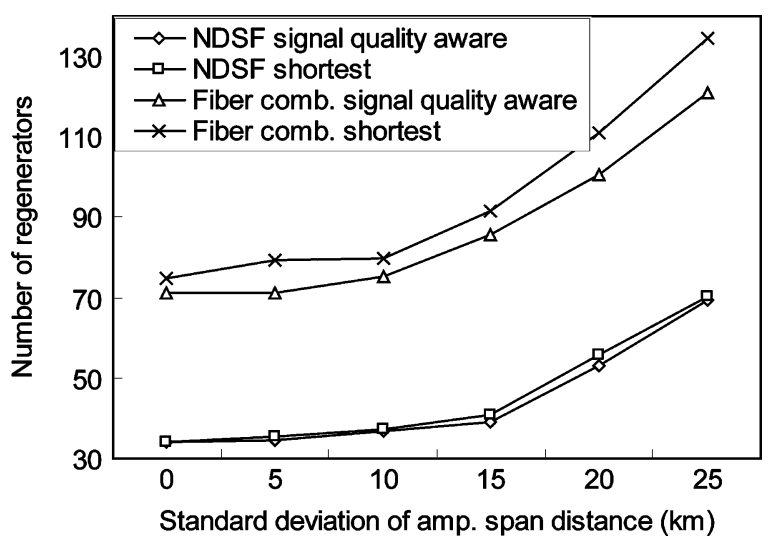

Fig. 4. Results of the large carrier network A with the fiber combination of $60 \%$ NDSF, $10 \%$ ELEAF, $10 \%$ TrueWave, $10 \%$ LS, and 10\% DSF, and a single NDSF fiber type.

\section{B. Result Analyses}

1) Impacts of Lightpath Routing Algorithms, Standard Deviation of Amplification Span Distances, and Fiber Types: We evaluate the impacts of lightpath routing algorithms, variances of amplification span distances, and fiber types on optical network design performance. For the network scenario with different types of fibers, we assume that the fiber type distribution is $60 \%$ NDSF, $10 \%$ ELEAF, $10 \%$ True Wave, $10 \%$ LS, and $10 \%$ DSF. For the network scenarios with the same fiber type, we assume that all the fiber links are NDSF.

Fig. 4 shows the results of the large carrier network A under the cases with combined fiber types and a single NDSF fiber type. The $x$ axis shows the standard deviation (std.) of amplification span distance and the $y$ axis shows the number of required regenerators (which also includes the number of additional wavelength converters for wavelength conversion). In the current simulation studies, due to low traffic load the number of required wavelength converters are found to be small since deployed signal generators can function as wavelength converters as well. Each curve point is an average of five network design scenarios, which are randomly generated under a certain span distance variance.

It is found that with the increase of span distance standard deviation, more regenerators are required. This is reasonable since a large standard deviation corresponds to a high non-uniformness of amplification span distance. A fiber link with long amplification spans generally shows a low OSNR, and a network with many these low-OSNR fiber links often requires more regenerators.

It is also found that the signal-quality-aware algorithm always requires fewer regenerators than the shortest path algorithm. This is attributed to the fact that the signal-quality-aware algorithm takes into account the physical-layer impairment information and can find a route that is expected to require the fewest regenerators. For the situation of combined fiber types, the improvement in terms of the average number of required regenerators is always greater than $4 \%$ and up to $10 \%$. For the single NDSF fiber type, the improvement is relatively smaller, ranging from $0 \%$ to $5 \%$. Moreover, comparing the results of these two

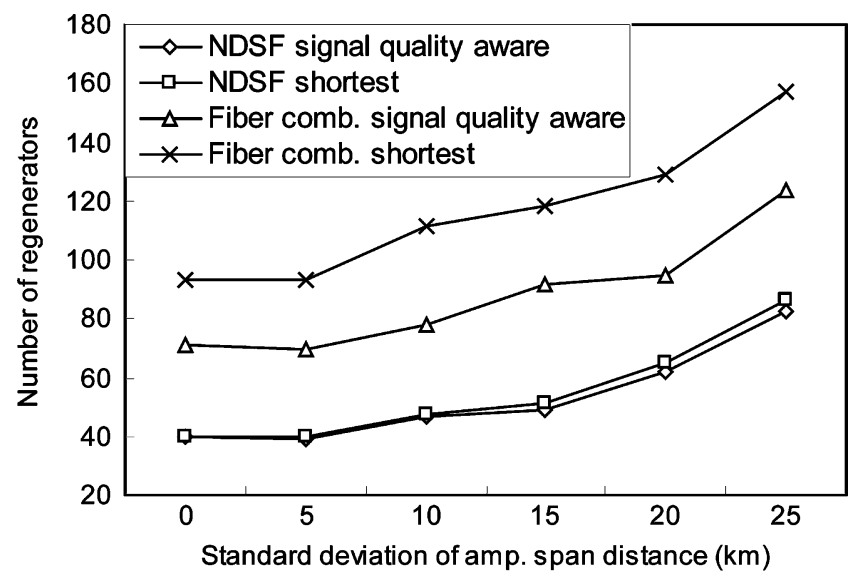

Fig. 5. Results of the USNET network with the fiber combination of $60 \%$ NDSF, $10 \%$ ELEAF, $10 \%$ True Wave, $10 \%$ LS, and $10 \%$ DSF, and a single NDSF fiber type.

network design cases, we find that the signal-quality-aware algorithm can bring more benefits to a network containing multiple fiber types. This is because most non-NDSF fibers in a network with the combined fiber types show lower OSNRs, which provides more opportunity for the signal-quality-aware routing algorithm to better select routes and minimize required regenerators by avoiding those low-OSNR links.

In addition, we would like to point out that the current results are specific to a certain set of national backbone design scenarios. It can be anticipated that the performance difference between the two routing algorithms can become smaller when fewer signal regenerators are required due to the reasons like a lower threshold OSNR, or a geographically smaller network. As an extreme case, in a metro-area network, which spans a very small region, we essentially do not need to deploy any signal regenerators, but simply employ the shortest path routing algorithm to find the best routes, since even the longest end-to-end lightpath in such a network does not require any signal regenerators.

Simulations were also performed for the USNET network. Fig. 5 shows the results under the situations of a combination of different fiber types and a single NDSF fiber type. Similar to the results of the large carrier network A, the signal-qualityaware algorithm always achieves better performance than that of the shortest-path algorithm. For the USNET network, the improvements are even larger. The average improvement is around $21 \%-30 \%$ for the case with combined fibers and $0 \%-5.5 \%$ for the case of NDSF fiber.

We also compare the results of the USNET network and the large carrier network A. It seems that the signal-quality-aware algorithm is more efficient for the former than the latter. The reason for this is that the USNET network has a better network connectivity than that of the large carrier network A. A good connectivity means more distinct routes between each pair of nodes, which provides more opportunity for the signal-quality-aware algorithm to select the best-signal-quality route, thereby achieving better performance. It can be concluded that an impairment-aware lightpath routing algorithm is more efficient for a network with a higher nodal degree. 


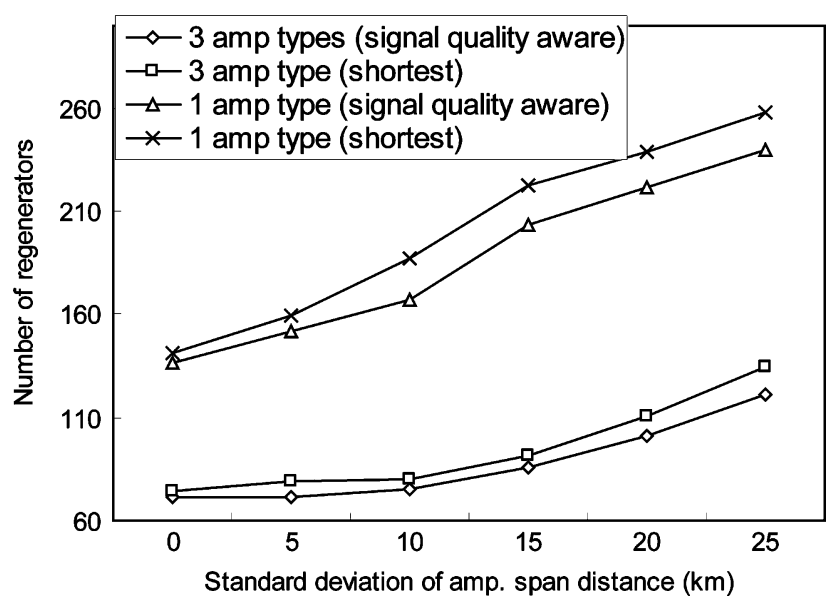

Fig. 6. Performance comparison between the cases of three amplification types and a single amplification type.

2) Impact of Supporting Amplifier Types: We also evaluate the impact of amplifier types on the network design performance. Specifically, in addition to the three amplifier types, we considered a single amplification type that is implemented as a combination of a 22-dB EDFA and a 7-dB Raman amplifier, and ensures to support all the amplification gain requirements. We ran simulations for the two amplification cases under the large network A with the combined fiber types.

Under different available amplification types, Fig. 6 compares the design performance in terms of number of required regenerators. It is clear to see that a network with more amplification options can achieve much better performance than that with a single amplification option. Specifically, we see that the improvement by the three amplification types over the single amplification type is very significant, up to $48 \%-58 \%$. This is because the former provides more options to select amplifiers that have the lowest NF (the best performance) for each amplification situation, while the latter does not have such a kind of flexibility and therefore can suffer from poor NFs in many amplification situations. Based on the results, we conclude that for an optical transport network with much physical-layer heterogeneity, it is necessary to design and deploy different types of amplifiers to cater for different amplification situations.

3) Node Regenerator Number Distribution: Another comparison is performed for the distribution of the number of regenerators on each network node. As a sample result, Fig. 7 shows the regenerator distribution of the USNET network under the combined fiber types and amplification span distance std. = $10 \mathrm{~km}$. It is interesting to see that the two algorithms show good correlation on the numbers of regenerators on the network nodes. The correlation coefficient ${ }^{1}$ of the case study is very high to be 0.97 . Similar observations are also found for the large carrier network A under other design scenarios with different amplification span distance stds. For example, for the large network A under the combined fiber types and amplification span distance std. $=10 \mathrm{~km}$, the correlation coefficient is even higher to

\footnotetext{
${ }^{1}$ The two algorithms find the required numbers of regenerators on each of the nodes, which form two node-based regenerator number distributions. The correlation coefficient is just computed based on these two distributions.
}

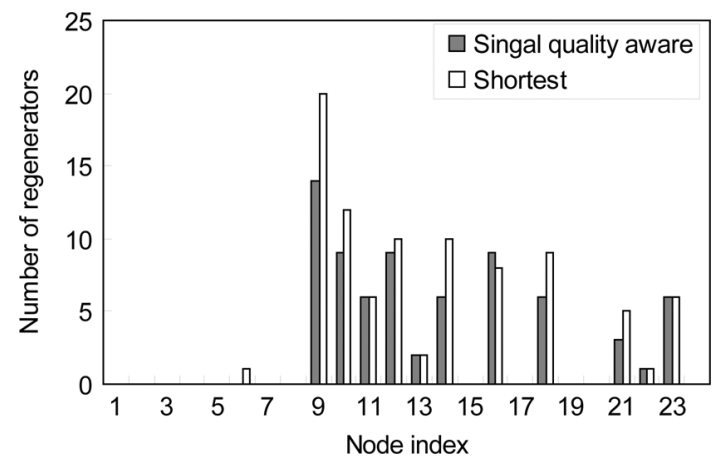

Fig. 7. Distribution of the number of regenerators on each network node (USNET with combined fiber types and amplification span distance std. = $10 \mathrm{~km})$.

be more than 0.99 . The high correlation coefficients imply that the distribution of required regeneration nodes in a network is likely independent of the routing algorithm applied, but is dependent on other network aspects such as topology, lightpath traffic demand distribution, and so on.

\section{CONCLUSION}

We developed an IA-RWA framework that takes into account various physical-layer heterogeneity, including variable amplification gain requirement, different amplifier types, and different fiber types. To reduce the required number of regenerators, we proposed a new routing algorithm, called the signal-quality-aware algorithm, to find a route that shows good signal quality between each node pair. A simple but efficient regenerator placement algorithm was applied to place regenerators on a lightpath. Also, the traditional first-fit wavelength assignment algorithm was extended to fully utilize the wavelength conversion capability of the placed regenerators.

Through the simulation studies, we evaluated the impacts from various perspectives, including lightpath routing algorithms, standard deviations of amplification span distances and attenuation coefficients, amplification types, as well as fiber types. We found that the proposed signal-quality-aware routing algorithm can consistently outperform the shortest-path routing algorithm to require fewer regenerators; the reduction is up to $10 \%-30 \%$ depending on specific network topologies and lightpath traffic demands. Also, the fiber types show a strong impact on the network design performance. It is found that the signal-quality-aware routing algorithm performs more efficiently when a network contains different fiber types. In addition, the evaluation on the impact of amplification span distance variance indicates that the signal-quality-aware routing algorithm performs better under a larger standard deviation of span distance. Also, the performance comparison of network designs with multiple amplifier types and a single amplifier type indicates that multiple amplification options are important for cost-effective network design, which can save up to more than 50\% signal regenerators over a single amplifier option. Finally, it is interesting to observe that the layout of regeneration nodes seems independent of applied routing algorithms, but dependent on lightpath demand matrix and network topology. 


\section{REFERENCES}

[1] I. Chlamtac, A. Ganz, and G. Karmi, "Lightpath communications: An approach to high bandwidth optical WAN's," IEEE Trans. Commun., vol. 40, no. 7, pp. 1171-1182, Jul. 1992.

[2] H. Zang, J. P. Jue, and B. Mukherjee, "A review of routing and wavelength assignment approaches for wavelength-routed optical WDM networks," Opt. Netw. Mag., pp. 47-60, Jan. 2000.

[3] G. Bernstein, Y. Lee, and W. Imajuku, "Framework for GMPLS and PCE control of wavelength switched optical networks (WSON)," IETF Draft, Draft-Ietf-CCAMP-Wavelength-Switched-Framework-01.txt.

[4] B. Ramamurthy, D. Datta, H. Feng, J. P. Heritage, and B. Mukherjee, "Impact of transmission impairments on the teletraffic performance of wavelength-routed optical networks," J. Lightw. Technol., vol. 17, no. 10, pp. 1713-1723, Oct. 1999.

[5] Y. Huang, J. P. Heritage, and B. Mukherjee, "Connection provisioning with transmission impairment consideration in optical WDM networks with high-speed channels," J. Lightw. Technol., vol. 23, no. 3, pp. 982-993, Mar. 2005.

[6] M. Gagnaire and S. A. Zahr, "Impairment-aware routing and wavelength assignment in translucent networks: State of the art," IEEE Commun. Mag., pp. 55-61, May 2009.

[7] K. Manousakis, K. Christodoulopoulos, E. Kamitsas, I. Tomkos, and E. A. Varvarigos, "Offline impairment-aware routing and wavelength assignment algorithms in translucent WDM optical networks," J. Lightw. Technol., vol. 27, no. 12, pp. 1866-1877, Jun. 2009.

[8] S. Pachnicke, T. Pashenda, and P. M. Krummrich, "Physical impairment based regenerator placement and routing in translucent optical networks," in Proc. OFC/NFOEC 2008, paper OWA2.

[9] T. Deng, S. Subramanian, and J. Xu, "Crosstalk-aware wavelength assignment in dynamic wavelength-routed optical networks," in Proc. 1st Int. Conf. Broadband Networks (BROADNETS), 2004.

[10] J. He, M. Brandt-Pearce, and S. Subramaniam, "QoS-aware wavelength assignment with BER and latency guarantees for crosstalk limited networks," in Proc. ICC, 2007, pp. 2336-2341.

[11] G. Shen, W. V. Sorin, and R. S. Tucker, "Cross-layer design of ASE-noise-limited island-based translucent optical networks," $J$. Lightw. Technol., vol. 27, no. 11, pp. 1434-1442, Jun. 2009.

[12] G. Shen, W. D. Grover, T. H. Cheng, and S. K. Bose, "Sparsely placement of electronic switching nodes for low blocking in translucent optical networks," J. Opt. Networking, vol. 1, no. 12, pp. 424-441, Dec. 2002.

[13] X. Yang and B. Ramamurthy, "Sparse regeneration in translucent wavelength-routed optical networks: Architecture, network design and wavelength routing," Photon. Netw. Commun., vol. 10, no. 1, pp. 39-53, Jan. 2005.

[14] G. Shen and R. S. Tucker, "Translucent optical networks: The way forward," IEEE Commun. Mag., pp. 48-54, Feb. 2007.

[15] S. Rumley and C. Gaumier, "Cost aware design of translucent WDM transport networks," in Proc. ICTON, 2009, pp. 1-4.

[16] S. Azodolmolky et al., "A dynamic impairment-aware networking solution for translucent mesh optical networks," IEEE Commun. Mag., pp. 38-47, May 2009.

[17] S. Pachnicke, N. Luck, and P. M. Krummrich, "Online physical-layer impairment-aware routing with quality of transmission constraints in translucent optical networks," in Proc. ICTON, 2009, paper Tu.A3.5.

[18] K. Katrinis, A. Tzanakaki, and G. Markidis, "Impairment-aware WDM network dimensioning with optimized regenerator placement," in Proc. OFC/NFOEC, 2009, paper NThE4.

[19] G. Shen and R. S. Tucker, "Sparse traffic grooming in translucent optical networks," J. Lightw. Technol., vol. 27, no. 20, pp. 4471-4479, Oct. 2009.

[20] N. Sambo et al., "Accounting for shared regenerators in GMPLS-controlled translucent optical networks," J. Lightw. Technol., vol. 27, no. 19, pp. 4338-4347, Oct. 2009.
[21] L. Wang, J. Zhang, G. Gao, Y. Liu, X. Chen, and W. Gu, "A hybrid control architecture for connection management in translucent WDM networks," in Proc. Globecom, 2008.

[22] S. Tibuleac and M. Filer, "Transmission impairments in DWDM networks with reconfigurable optical add-drop multiplexers," J. Lightw. Technol., vol. 28, no. 4, pp. 557-568, Feb. 2010.

[23] S. Pachnicke, T. Paschenda, and P. M. Krummrich, "Assessment of a constraint-based routing algorithm for translucent $10 \mathrm{Gbits} / \mathrm{s}$ DWDM networks considering fiber nonlinearities," J. Opt. Networking, vol. 7, no. 4, pp. 365-377, Apr. 2008.

Gangxiang Shen (S'98-M'99) received the B.Eng. degree from Zhejiang University, Hangzhou, China, the M.Sc. degree from Nanyang Technological University, Singapore, and the Ph.D. degree in electrical and computer engineering from the University of Alberta, Edmonton, AB, Canada, in 2006.

$\mathrm{He}$ is a Distinguished Professor with School of Electronic and Information Engineering, Soochow University, China. Before he joined Soochow University, he was Lead Engineer with Ciena Corporation, Linthicum, MD. He was also an Australian ARC Postdoctoral Fellow with the ARC Special Research Centre for Ultra-Broadband Information Networks, Department of Electrical and Electronic Engineering, University of Melbourne, Melbourne, Australia. He is an editorial board member of the Optical Switching and Networking. His research interests are in spectrum efficient optical networks, green optical networks, and integrated wireless and optical networks. He has authored or coauthored more than 45 peer-reviewed technical papers.

Prof. Shen is a Lead Guest Editor of the IEEE Journal ON SELECTED AREAS IN COMMUNICATIONS Special Issue of "Next-Generation Spectrum-Efficient and Elastic Optical Transport Networks." He served as TPC members and workshop chairs for various international conferences such as INFOCOM, GLOBECOM, and ICC. He received Young Researcher New Star Scientist Award in "2010 Scopus Young Researcher Award Scheme" in China. He was a recipient of the Izaak Walton Killam Memorial Award from the University of Alberta, and the Canadian NSERC Industrial R\&D Fellowship.

Yunfeng Shen (M'02) was born in Henan Province, China, in 1973. He received the Bachelor degree in telecommunication and control engineering from the Northern Jiaotong University and the Ph.D. degree in electromagnetic field and microwave technology from the Beijing University of Posts and Telecommunications, Beijing, China, in 1994 and 1999, respectively.

He had been with the Lightwave department in Ciena Corporation since 2001. Currently he is leading the system development team for optical design tools. From 1999 to 2000, he was a research fellow at the Nanyang Technological University, Singapore. His current research interests include simulation and modeling of high speed optical fiber communication systems, and the design and planning tools of optical networks. He has authored over 30 technical papers in the fields of optical fiber communications.

Harshad P. Sardesai was born in Mumbai, India. He received the B.E. degree from the University of Bombay, Mumbai, India, the M.S. degree from the University of Texas at Arlington, and the Ph.D. degree from Purdue University, West Lafayette, IN, all in electrical engineering.

Since 1997, he has been with Ciena Corporation, Linthicum, MD, where he currently is in charge of customer facing network design and planning tools, across all network layers from transport to switching to Ethernet. He previously worked in the transport layer contributing to Ciena's first 10-Gb/s transport system, and then to Ciena's $40-$ and 100-Gb/s transport product development. Prior to his current responsibilities, he was Director of Lightwave Systems group where his staff was responsible for architecture, design, and testing of Ciena's transport solutions. He has over 40 technical publications and holds 13 U.S. patents. 